\title{
Amplification of ultrafast pulses in an extended Mamyshev regenerator
}

Repgen, Paul, Wandt, Dieter, Morgner, Uwe, Neumann, Jörg, Kracht, Dietmar

Paul Repgen, Dieter Wandt, Uwe Morgner, Jörg Neumann, Dietmar Kracht, "Amplification of ultrafast pulses in an extended Mamyshev regenerator," Proc. SPIE 11260, Fiber Lasers XVII: Technology and Systems, 112600P (21 February 2020); doi: 10.1117/12.2546176

SPIE. Event: SPIE LASE, 2020, San Francisco, California, United States 


\title{
Amplification of ultrafast pulses in an extended Mamyshev regenerator
}

\author{
Paul Repgen ${ }^{\mathrm{a}}$, Dieter Wandt ${ }^{\mathrm{a}}$, Uwe Morgner ${ }^{\mathrm{a}, \mathrm{b}, \mathrm{c}}$, Jörg Neumann ${ }^{\mathrm{a}, \mathrm{c}}$, and Dietmar Kracht ${ }^{\mathrm{a}, \mathrm{c}}$ \\ a'Laser Zentrum Hannover, Hollerithallee 8, 30419 Hannover, Germany \\ bInstitut für Quantenoptik, Leibniz Universität Hannover, Welfengarten 1, 30167 Hannover, \\ Germany \\ ${ }^{\mathrm{c}}$ Cluster of Excellence PhoenixD (Photonics, Optics, and Engineering Innovation Across \\ Disciplines), Hannover, Germany
}

\begin{abstract}
We present the characteristics of a high-energy ultrafast Yb-fiber laser system, based on a Mamyshev oscillator and a subsequently arranged fiber amplifier stage. The Mamyshev oscillator emits pulses at a repetition rate of $11 \mathrm{MHz}$ and pulse energies of $31 \mathrm{~nJ}$. These pulses are spectrally filtered and amplified in a Yb-doped fiber up to $1 \mu \mathrm{J}$ pulse energy which could be temporally dechirped to less than 50 fs autocorrelation duration. We discuss the scaling as well as limiting options related to pulse energies and duration.
\end{abstract}

Keywords: Mamyshev oscillator, nonlinear amplifier, femtosecond fiber laser

\section{INTRODUCTION}

High power ultrafast laser systems operating around a wavelength of $1 \mu \mathrm{m}$ are widely used light sources in many industrial applications, such as micro-machining and processing of metals, ceramics, glasses etc. or imaging applications like nonlinear microscopy. These systems are usually based on a low power master oscillator, emitting pulse energies of a few nanojoule, followed by one or more amplifier stages, to generate the necessary output parameters. However, during amplification, the pulse parameters of the oscillator can be altered, e.g., the spectral width of the emission can be reduced due to gain narrowing, which increases the minimum possible pulse duration, or the pulse shape can be distorted by an excessively accumulated nonlinear phase. Recently, a design termed Mamyshev oscillator (MO) has attained a lot of attention, owing to its generation of modelocked laser pulses with outstanding output parameters in terms of output energy, spectral bandwidth, and pulse duration. The mode-locking mechanism in a MO relies on offset spectral filtering between the sections of spectral regeneration by self-phase modulation (SPM) in optical fibers, so that only intense, spectrally broadened pulses are transmitted by the following filter. Depending on the spectral separation of the filters, this saturable absorption can generate a strong modulation depth, leading to a very high tolerance of nonlinear phase in the resonator of more than $60 \pi{ }^{1}$ Subsequently, higher output pulse energies compared to other laser configurations can be generated. Small spectral filter separations in the range of $\sim 1 \mathrm{~nm}$ with overlapping transmission spectra cause rather low modulation depths, rendering the design of self-starting Mamyshev oscillators, reported by Regelskis et al. ${ }^{2}$ or by Tarasov et al. ${ }^{3}$. Increased filter separations, i.e., in the range of $\sim 10 \mathrm{~nm}$ to $\sim 25 \mathrm{~nm}$, enable the generation of extremely broad spectra, corresponding to pulse durations in the sub-50 fs regime, as reported by Liu et al. ${ }^{4}$. Extremely high pulse energies of $1 \mu \mathrm{J}$ could be achieved according to Liu et al. ${ }^{5}$ by using large-core photonic crystal fibers in the MO. However, due to the large filter separation, these setups only reproduce injected pulses, and the mode-locking cannot start from noise.

In this paper, we present a Mamyshev oscillator-amplifier system as well as investigations on the scalability of this layout in terms of output pulse energy, combined with very short pulse durations. Accordingly, we extended the concept of the MO by a corresponding fiber amplifier stage, consisting of step-index few-mode fibers and studied the performance, depending on several input parameters, like spectral width and spectral shape of the input pulse.

Further author information: (Send correspondence to P. Repgen)

P. Repgen: E-mail: p.repgen@lzh.de

Fiber Lasers XVII: Technology and Systems, edited by Liang Dong, Proc. of SPIE Vol. 11260, 112600P

(C) $2020 \mathrm{SPIE} \cdot$ CCC code: 0277-786X/20/\$21 · doi: 10.1117/12.2546176 


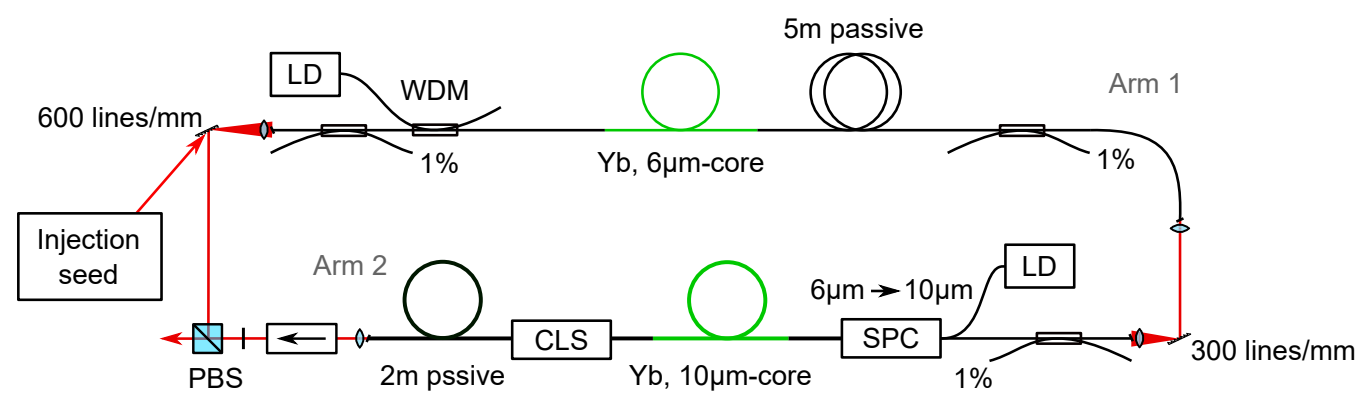

Figure 1. Setup scheme of the Mamyshev oscillator. CLS: cladding light stripper, LD: Pump laser diode, PBS: Polarizing beam splitter cube, SPC: Signal-pump combiner, WDM: Wavelength division multiplexer.

\section{EXPERIMENTS AND DISCUSSION}

The setup of our MO is presented in Figure 1, as also reported in reference $\left.{ }^{6}\right]$. It was set up as a ring resonator with two amplification stages, separated by band-pass filters (BPFs). In the first amplification stage, an injected seed pulse was amplified in a single-mode, single-clad amplifier, core-pumped by a laser diode with a maximum power of $700 \mathrm{~mW}$ at a wavelength of $976 \mathrm{~nm}$. After amplification, the pulse was spectrally shaped in a $5 \mathrm{~m}$-section of passive single-mode fiber and afterwards coupled out. The BPF, separating the first and second amplifier, was a reflective diffraction grating with 300 lines $/ \mathrm{mm}$, transmitting a Gaussian spectrum with a spectral width of $2.1 \mathrm{~nm}$. In the second amplification stage, the pulse was amplified in a double-clad fiber with a $10 \mu \mathrm{m}$ corediameter. The active fiber was pumped by a multimode pump diode with a maximum power of $10 \mathrm{~W}$. The residual pump light behind the active fiber was stripped by a cladding light stripper (CLS). A 2 m-long section of passive fiber supported the spectral shaping by SPM behind the amplifier. A free-space isolator ensured uni-directional propagation. Behind the isolator, roughly $95 \%$ of the circulating pulse energy was coupled out by a polarizing beam splitter cube (PBS). All fibers and fiber components were polarization maintaining (PM).

The MO reproduced a stable pulse train with an output power of $331.5 \mathrm{~mW}$ at a repetition rate of $10.84 \mathrm{MHz}$, corresponding to $31 \mathrm{~nJ}$ pulse energy. The optical and the radio-frequency spectrum of the MO are presented in Figure 2 (a) and (b), respectively. For a spectral separation of the BPFs of $10 \mathrm{~nm}$, the main part of the output spectrum spanned from $1000 \mathrm{~nm}$ to $1080 \mathrm{~nm}$ and showed a sidelobe at a maximum wavelength of around $1110 \mathrm{~nm}$. This side maximum is believed to result from stimulated Raman scattering. The radio-frequency trace showed a signal-to-noise ratio of $76 \mathrm{~dB}$, proving stable mode-locking.

For different applications, like in material processing, the output pulse energy of $31 \mathrm{~nJ}$ is not sufficient and
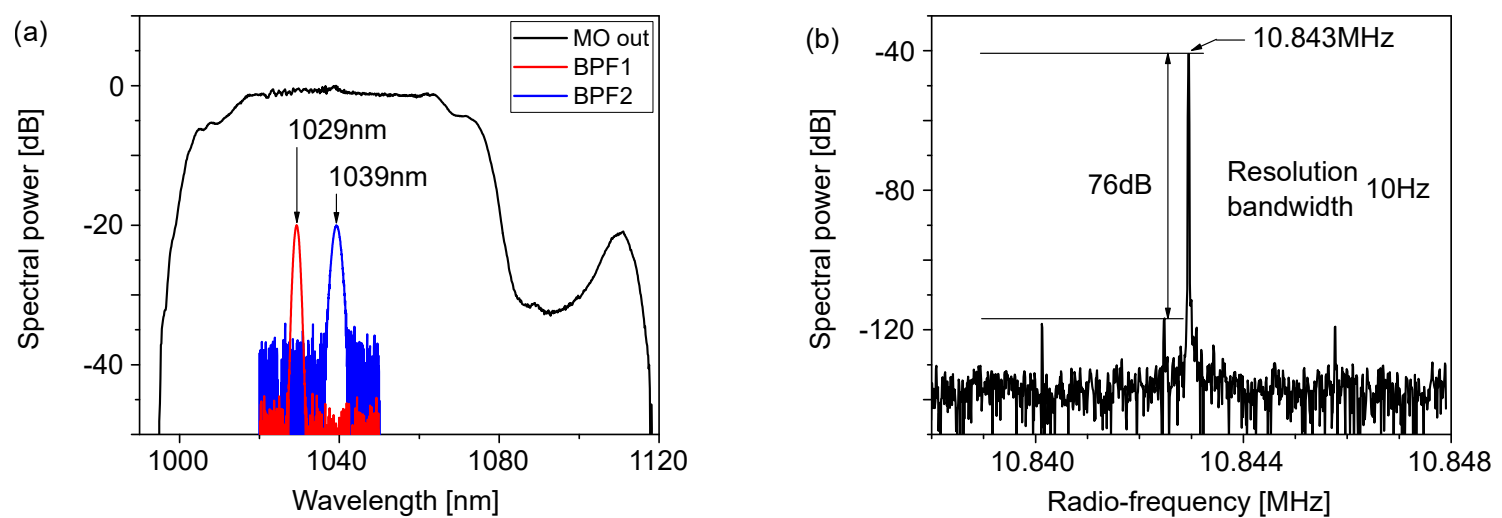

Figure 2. (a) the optical spectrum of the Mamyshev oscillator output and the transmission spectrum of the band-pass filters, (b) the fundamental peak in the radio-frequency spectrum in the mode-locked state of the Mamyshev oscillator with a span of $10 \mathrm{kHz}$. 


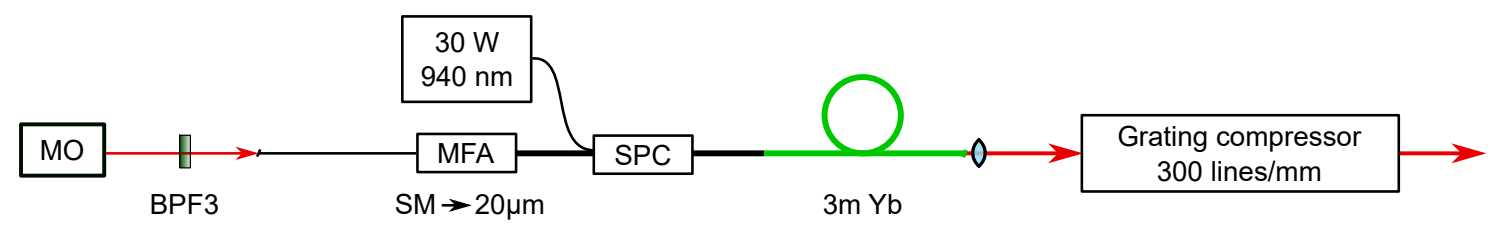

Figure 3. Setup of the power amplifier stage. BPF3: Band-pass filter, MFA: Mode-field adapter, MO: Mamyshev oscillator, SM: Single-mode, SPC: Signal-pump combiner.

needs to be amplified. This is usually achieved by chirped pulse amplification (CPA), i.e. temporally stretching the pulse before amplification and recompressing it afterwards. This process has limitations owing to a low tolerance to nonlinear phase of more than $\pi$ and the reduction of spectral width due to gain narrowing. ${ }^{7,8}$ On the other hand, mimicking the oscillator in the amplification stage offers extremely promising results. ${ }^{9}$ Therefore, the power amplifier was designed like one of the arms in the MO, as depicted in Figure 3. The pulses were spectrally filtered and then coupled into a single-mode fiber to ensure guidance of the fundamental mode. In order to exploit parabolic pulse evolution in the amplifier and reduce the influence of nonlinear effects (to allow for higher power), the active fiber had a length of $3 \mathrm{~m}$ and a core diameter of $20 \mu \mathrm{m}$ (nlight, Yb800-20/125DC-PM). It was pumped by a multi-mode laser diode with a maximum power of $30 \mathrm{~W}$ at a wavelength of $940 \mathrm{~nm}$. Low doping concentration of the active fiber and pumping at a rather inefficient wavelength ensured a low gain along the fiber, supporting the parabolic pulse evolution. ${ }^{10}$ The mode-field of the amplifier fiber and the single-mode fiber were adapted by a fiber mode-field adapter. A passive fiber behind the amplification to support spectral broadening by SPM was not necessary. After amplification, the pulses were stretched to a duration of roughly $10 \mathrm{ps}$ and could be compressed by a grating compressor with grating line densities of 300 lines $/ \mathrm{mm}$.

Next to amplification of the pulses from the MO, this design could also be used to study the performance and scalability of the MO, owing to the similiarity of the power amplifier and the MO arms. For example the choice of a fitting BPF in front of the amplifier, referred to as BPF3, was crucial. Due to the second order dispersion on the pulses from the $\mathrm{MO}$, the temporal pulse shape in the power amplifier depended on the spectral transmission shape of BPF3. We tested interference BPF3s with $3 \mathrm{~nm}$ and $5 \mathrm{~nm}$ bandwidth at a central wavelength of $1030 \mathrm{~nm}$ and $1040 \mathrm{~nm}$ and an interference BPF3 with a spectral bandwidth of $10 \mathrm{~nm}$ at $1030 \mathrm{~nm}$ and $1060 \mathrm{~nm}$ with an almost arbitrary spectral transmission shape. Furthermore, we used a diffraction grating with $300 \mathrm{lines} / \mathrm{mm}$ and one with 600 lines $/ \mathrm{mm}$ at central wavelengths of $1040 \mathrm{~nm}$ and $1060 \mathrm{~nm}$. The diffraction gratings generated a Gaussian transmission shape.

\subsection{Filtering with interference band-pass filters}

The results of the amplification after filtering with different interference BPF3s are collected in Figure 4. In particular, the input spectrum and the spectrum for the maximum possible amplification are shown, as well as the autocorrelation (AC) trace of the compressed pulse with maximum power.

When using narrow-band interference BPFs with a transmission width of $3 \mathrm{~nm}$ or $5 \mathrm{~nm}$, the pulse evolution in the amplifier was comparable. The pulses could not be strongly amplified for a central wavelength of $1030 \mathrm{~nm}$ (Fig. 4 (a) and (e)). At an amplified signal above $1.2 \mathrm{~W}$ and $0.4 \mathrm{~W}$ for the $3 \mathrm{~nm}$-filter and the $5 \mathrm{~nm}$-filter, respectively, self-lasing occurred in the power amplifier. This problem could be circumvented by increasing the central wavelength of the input to $1040 \mathrm{~nm}$ (Fig. 4 (c) and (g)). In this case, the maximum output power was $5.2 \mathrm{~W}$ and $5.8 \mathrm{~W}$, respectively. As can be seen in the AC traces, the pulse quality severely degraded at these output powers. The degradation resulted from the excessive amount of SPM on the pulses. The qualitative shape of the SPM-induced frequency chirp $\delta \omega$ is the derivative of the nonlinear phase $\phi_{\mathrm{NL}}$ with respect to time, ${ }^{11}$

$$
\delta \omega(T)=-\frac{\partial \phi_{\mathrm{NL}}}{\partial T}
$$

The transmitted spectra of the interference BPFs have sharp edges and a plateau in the center, comparable to a super-Gaussian shape. Therefore, the strong frequency chirp induced by the steep edges, generated a 

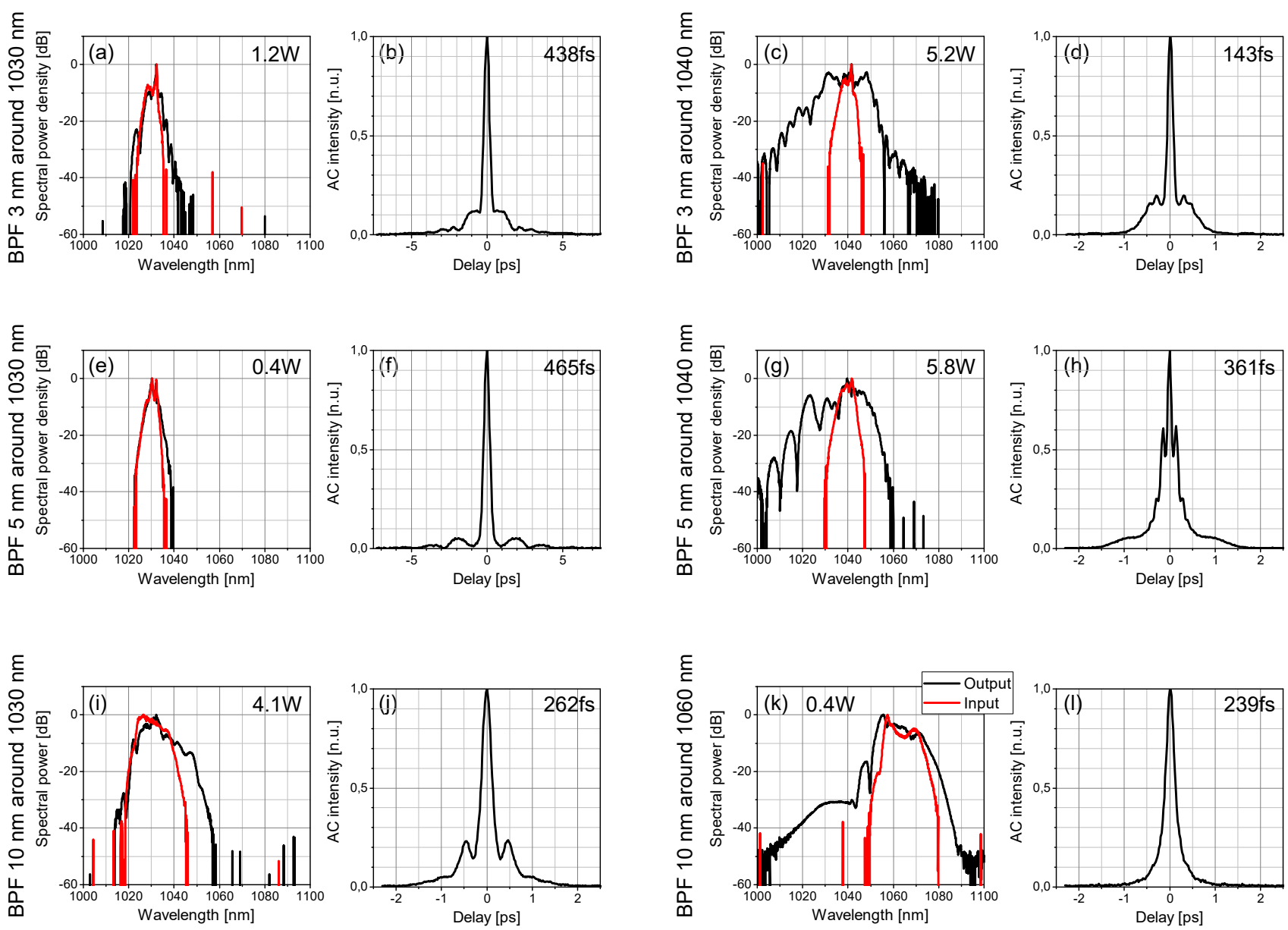

Figure 4. Results of the amplification after filtering with band-pass filters (BPFs). In first and third column, the input spectra (red) and the output spectra at the highest amplification value (black) are shown for different interference bandpass filters in front of the power amplifier. In the second and forth column, the corresponding autocorrelation trace at the highest output power are shown.

broad optical spectrum during amplification. But the induced frequency chirp is not linear and cannot be easily compressed by the grating compressor, hence producing strong sidebands in the AC traces.

Compared to the small-bandwidth filters, the pulse evolution by using a $10 \mathrm{~nm}$-BPF as BPF3 was different. The pulses could be amplified to $4.1 \mathrm{~W}$ output power for the central wavelength around $1030 \mathrm{~nm}$ (Fig. 4 (i)). At higher pump powers, nonlinear pulse breaking occurred. Because of the higher input pulse energy resulting from the broader spectrum, a high amount of nonlinear phase can already be accumulated in the passive fiber section in the beginning of the amplifier. Nonlinear pulse breaking could be verified from the AC trace. A broad pedestal with a coherence spike was formed.

The pulse evolution after the filtering by using BPF3 with the $10 \mathrm{~nm}$ transmission spectrum and with a center wavelength of $1060 \mathrm{~nm}$ was also tested (Fig. $4(\mathrm{k})$ ). In this case, the pulses could not be amplified to more than $0.4 \mathrm{~W}$ output power, as excessive amplified spontaneous emission occurs around the maximum of the gain cross-section of Yb-doped fibers. Due to the low gain cross-section of $\mathrm{Yb}$ around $1060 \mathrm{~nm}$, amplified spontaneous emission (ASE) around $1030 \mathrm{~nm}$ is a severe problem in the amplifier. The spectral broadening by SPM and, therefore, the shift of the pulse spectrum, would reduce the amount of ASE, as can be seen when using diffraction gratings as BPF3, as described below. The pulse in the case from Figure $4(\mathrm{k})$ and (l), however, is not strong enough to generate sufficient new wavelength components to compete with ASE. 


\subsection{Filtering with diffraction gratings}

Using a diffraction grating as BPF3 yielded a wavelength spectrum with a Gaussian shape. The results of the amplification with this filter type are presented in Figure 5. We used a 600 lines $/$ mm-grating (Fig. 5 (a), (e), (b) and (f)) and a 300 lines/mm-grating (Fig. 5 (c), (g), (d) and (h)) at center wavelengths of $1040 \mathrm{~nm}$ and $1060 \mathrm{~nm}$.

The highest output pulse energy was achieved for the amplifier setup with a 600 lines/mm-grating as BPF3 and at a central input wavelength of $1040 \mathrm{~nm}$ and a spectral width of $1.3 \mathrm{~nm}$, presented in Figure 5 (a) and (e). With these parameters, the pulse energy could be increased to more than $1 \mu \mathrm{J}$, corresponding to $11.2 \mathrm{~W}$ average power at a repetition rate of $10.84 \mathrm{MHz}$. The spectral width increased monotonically for increasing pump power, as expected from the parabolic pulse evolution. The Fourier-transformed pulse duration was $39 \mathrm{fs}$, despite of the rather prominent peak at the wavelength $1040 \mathrm{~nm}$. The Fourier-transformed pulse duration is close to the measured AC duration of 63 fs (Fig. 5 (e)). The radio-frequency traces of the amplified signal did not show any degradation, therefore, self-lasing could be excluded. Interferometric ACs were taken, which showed a 1:8-ratio of the signal, indicating, that the measured AC trace (Fig. 5 (e)) is not just a coherence spike. ${ }^{6}$ Nonlinear distortions of the pulse in this amplifier setup were not observed. For higher output power than $11.2 \mathrm{~W}$, the signal power slowly decreased with increasing pump power. This is usually a sign of thermal mode instability (TMI). ${ }^{12}$

The maximum possible output pulse energy of more than $1 \mu \mathrm{J}$ could not be reached with the other filter parameters of the BPF3 which were investigated. At the different input wavelength of $1060 \mathrm{~nm}$ (Fig. 5 (b), (f)) and a spectral width of $1.5 \mathrm{~nm}$, the onset of TMI occurred at a pulse energy of $507 \mathrm{~nJ}$, corresponding to $5.5 \mathrm{~W}$ average power. Owing to the input wavelength and the gain cross-section of Yb-doped fibers, new wavelength components were generated preferably in the blue edge of the spectrum. Furthermore, the rapid amplification of the new wavelength components resulted in a relatively broad spectrum at low pulse energies, compared to the case from Figure 5 (a). Still, in this setup, nonlinear effects, like excessive SPM with a nonlinear spectral phase or pulse breaking, did not distort the pulse. The spectrum allows the generation of pulses with a Fourier-limited duration of $39 \mathrm{fs}$ and a Fourier-limited AC duration of $57 \mathrm{fs}$. This is still comparable to the measured AC duration of $81 \mathrm{fs}$, aside of uncompressable features due to the deviation from ideal parabolic pulse evolution. Deviations could result from the strong blue-shift due to the Yb-gain cross-section.
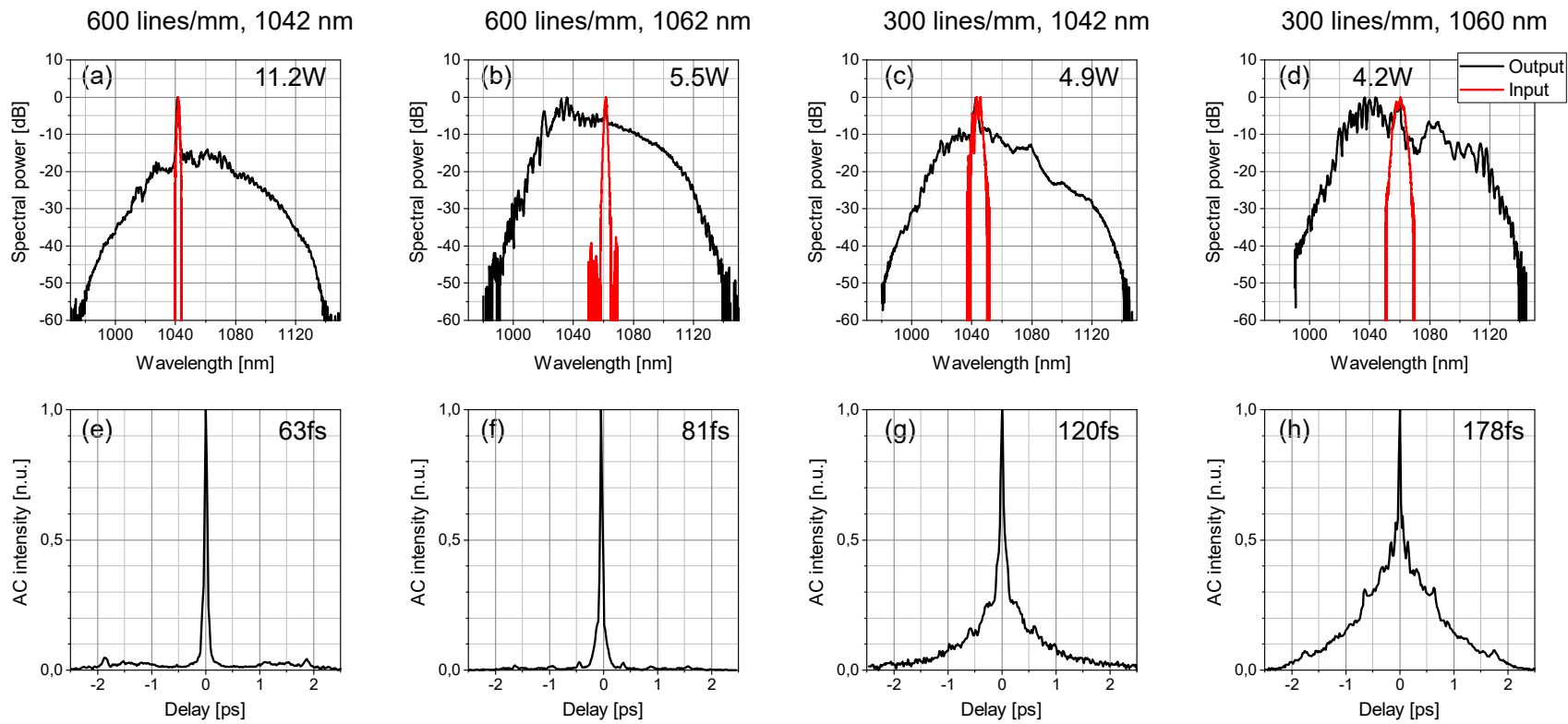

Figure 5. Results of the amplification after filtering with diffraction gratings as band-pass filter. The input spectrum (red) and the spectrum at the highest possible output power (black) are depicted in (a) - (d), the corresponding (compressed) autocorrelation traces are shown in (e) - (f). 
The amplification of larger spectral bandwidths by using a 300 lines/mm-grating as BPF3, was limited by nonlinear pulse breaking, independent of the central input wavelength. The spectral width was $4.0 \mathrm{~nm}$ and $5.3 \mathrm{~nm}$ for the central wavelength of $1042 \mathrm{~nm}$ (Fig. 5 (c)) and $1060 \mathrm{~nm}$ (Fig. 5 (d)), respectively. This is comparable to the results of the interference BPF with the $10 \mathrm{~nm}$ bandwidth, from Section 2.1 or the immediate amplification of an unchirped, high-energy pulse. It seems that the pulse accumulated too much nonlinear phase in the passive fiber section in the beginning of the power amplifier. The pulse breaking could be observed in the AC trace, by the forming of a parabolic pedestal with a coherence spike.

\section{SUMMARY}

We presented a Mamyshev oscillator-amplifier system in which the pulses from the oscillator could be amplified to more than $1 \mu \mathrm{J}$ and afterwards compressed to the 50 fs-range in a single amplifier stage, consisting of standard fibers. By designing the amplifier like one of the oscillator arms, the pulse evolution from the Mamyshev oscillator could be mimicked and large spectral bandwidths could be achieved. Furthermore, due to the similar setup, the amplification could be investigated depending on the input spectrum of the power amplifier. Different spectral shapes of the filtered pulse were achieved by using interference band-pass filters and diffraction gratings at different central input wavelengths. Best results were achieved with a narrow input spectrum at a wavelength close to the maximum of the gain cross-section of Ytterbium-doped fibers. A small spectral offset, however, is beneficial for the amplification as it prevented self-lasing of the power amplifier.

The results of the amplifier can be used to design a Mamyshev oscillator capable of emitting pulses in the $1 \mu \mathrm{J}$-range, consisting of standard step-index optical fibers.

\section{ACKNOWLEDGMENTS}

This project was funded by the German Federal Ministry of Education and Research (BMBF) under contract number 01DL17002A "OPUMAC".

\section{REFERENCES}

[1] Fu, W., Wright, L. G., Sidorenko, P., Backus, S., and Wise, F. W., "Several new directions for ultrafast fiber lasers [Invited]," Optics Express 26, 9432 (apr 2018).

[2] Regelskis, K., Želudevičius, J., Viskontas, K., and Račiukaitis, G., "Ytterbium-doped fiber ultrashort pulse generator based on self-phase modulation and alternating spectral filtering," Optics Letters 40, 5255 (nov 2015).

[3] Tarasov, N., Perego, A. M., Churkin, D. V., Staliunas, K., and Turitsyn, S. K., "Mode-locking via dissipative Faraday instability," Nature Communications 7, 12441 (aug 2016).

[4] Liu, Z., Ziegler, Z. M., Wright, L. G., and Wise, F. W., "Megawatt peak power from a Mamyshev oscillator," Optica 4, 649 (jun 2017).

[5] Liu, W., Liao, R., Zhao, J., Cui, J., Song, Y., Wang, C., and Hu, M., "Femtosecond Mamyshev oscillator with 10-MW-level peak power," Optica 6, 194 (feb 2019).

[6] Repgen, P., Wandt, D., Morgner, U., Neumann, J., and Kracht, D., "Sub-50 fs, J-level pulses from a Mamyshev oscillatoramplifier system," Optics Letters 44, 5973 (dec 2019).

[7] Perry, M. D., Ditmire, T., and Stuart, B. C., "Self-phase modulation in chirped-pulse amplification," Optics Letters 19, 2149 (dec 1994).

[8] Hotz, D. F., "Gain Narrowing in a Laser Amplifier," Applied Optics 4, 527 (may 1965).

[9] Pavlov, I., Rybak, A., Cenel, C., and Ilday, F. O., "Balancing gain narrowing with self phase modulation: 100-fs, 800-nJ from an all-fiber-integrated Yb amplifier," in [2013 Conference on Lasers 83 Electro-Optics Europe 8 International Quantum Electronics Conference CLEO EUROPE/IQEC], 927, 1-1, IEEE (may 2013).

[10] Fermann, M. E., Kruglov, V. I., Thomsen, B. C., Dudley, J. M., and Harvey, J. D., "Self-Similar Propagation and Amplification of Parabolic Pulses in Optical Fibers," Physical Review Letters 84(26), 6010-6013 (2000).

[11] Agrawal, G. P., "Effect of intrapulse stimulated Raman scattering on soliton-effect pulse compression in optical fibers," Optics Letters 15, 224 (feb 1990).

[12] Scarnera, V., Ghiringhelli, F., Malinowski, A., Codemard, C. A., Durkin, M. K., and Zervas, M. N., "Modal instabilities in high power fiber laser oscillators," Optics Express 27, 4386 (feb 2019). 